Voix et Images

voixetimages

\title{
Entrevue avec Yves Beauchemin
}

\section{Frances J. Summers}

Volume 12, numéro 3 (36), printemps 1987

Yves Beauchemin

URI : https://id.erudit.org/iderudit/200651ar

DOI : https://doi.org/10.7202/200651ar

Aller au sommaire du numéro

Éditeur(s)

Université du Québec à Montréal

ISSN

0318-9201 (imprimé)

1705-933X (numérique)

Découvrir la revue

Citer ce document

Summers, F. J. (1987). Entrevue avec Yves Beauchemin. Voix et Images, 12(3),

360-375. https://doi.org/10.7202/200651ar

Ce document est protégé par la loi sur le droit d'auteur. L'utilisation des services d'Érudit (y compris la reproduction) est assujettie à sa politique d'utilisation que vous pouvez consulter en ligne.

https://apropos.erudit.org/fr/usagers/politique-dutilisation/
Cet article est diffusé et préservé par Érudit.

Érudit est un consortium interuniversitaire sans but lucratif composé de l’Université de Montréal, l’Université Laval et l'Université du Québec à Montréal. Il a pour mission la promotion et la valorisation de la recherche. https://www.erudit.org/fr/ 


\title{
Entrevue avec Yves Beauchemin *
}

\author{
par Frances J. Summers, Canberra, Australie
}

V. \& I. - Vous avez dit au Soleil (6 juin 1981, p. E-3) que ce roman n'est pas un livre de divertissement. Alors, quel en est le but principal?

Y. Beauchemin - Votre question est un peu... En anglais on pourrait dire tricky. Embêtante, disons. N'importe quelle œuvre d'art cherche, en général, à créer le plaisir. Le contraire du plaisir, c'est la douleur ou l'ennui. Le mot "divertissement» figure sûrement parmi les antonymes du mot "ennui". Donc, bien sûr, j'ai cherché à divertir les gens, mais il y a une différence entre divertissement superficiel et plaisir profond. Si j'ai mis sept ans à écrire ce livre-là, c'est que je voulais procurer aux gens autre chose qu'un plaisir superficiel. On peut prendre du plaisir à lire un article dans Croc, ou un roman d'aventures superficiel qui nous aide à traverser quelques heures de déprime ou d'ennui. Mais le plaisir qu'on prend à lire les Frères Karamazov ou la Cousine Bette ou les Hommes de bonne volonté est d'un tout autre calibre. C'est vers ce genre d'œuvres que je tends spontanément, même si je sais fort bien qu'elles me dépassent. Je n'ai aucun intérêt pour l'écriture dite alimentaire ou commerciale. Mes modèles, donc, ce sont les grands écrivains que j’ai lus, dont certains ont modifié mon attitude face à la vie. Le plaisir que je cherche comme auteur et comme lecteur, c'est un plaisir capable de modifier la vie.

\section{V. \& I. - Avez-vous un message à transmettre dans le Matou?}

Y. Beauchemin - Je ne crois pas au roman-message. J'ai voulu écrire une histoire. J'ai voulu faire une cuvre d'art, quoi. Ma sœur, qui est peintre et qui a fait l'illustration de la couverture, a voulu tout simplement créer de la beauté. En écrivant le livre, c'est ce que j'ai essayé de faire, moi aussi. Ce n'est pas un roman à thèse; je n'avais pas d'idées derrière la tête lorsque je l'ai écrit. Je voulais tout simplement essayer de transmettre des émotions à un lecteur par le moyen d'une histoire et de personnages que j'essayais de rendre les plus intéressants possible. Bien sûr, quand je relis le livre, j'y retrouve des idées qui me tiennent à cœur. Mais ça s'est fait un peu malgré moi. Par exemple, on a relevé, dans le Matou, beaucoup de scènes de démolition à Montréal. Depuis la fin des années 70 , je vis sous l'effet d'un choc terrible. Je me suis aperçu à un moment donné que la ville que j'aimais tant me filait

* La présente entrevue a été réalisée en septembre 1984. 
entre les doigts: Drapeau était en train de la démolir. Il est très avancé, vous savez. Il est en train de détruire notre mémoire collective et historique. Il a poussé dans le néant une grande partie du paysage urbain montréalais, une multitude de beaux vieux édifices, de sections de rues, de perspectives pittoresques qui, chaque jour, nous rendaient heureux sans même que nous le sachions. Et dire qu'il s'apprête après ce long massacre à célébrer le $350^{\mathrm{e}}$ anniversaire de la fondation de Montréal... Quel cynisme! Ou quelle inconscience! C'est ce qu'on fait un peu partout en Amérique, un peu partout dans le monde. J'ai pris tout à coup conscience que la civilisation dans laquelle nous vivons n'a plus de cœur, plus de mémoire; elle a perdu le sens de la beauté et de la douceur de vivre en général, obsédée qu'elle est par l'efficacité - une sorte d'efficacité monstrueuse parfois - et le profit. L'architecture actuelle des villes modernes - il y a des tas d'exceptions, bien sûr, et bien des réussites - est soit tout à fait insignifiante et sans imagination (je l'appelle parfois de l'architecture anorexique), soit oppressive, inhumaine, nazie, quoi, Cette situation me tourmante et me fait souffrir. On sentira ces tourments dans mon troisième livre*. C'est une agression impardonnable, qui me révolte. Il y a également d'autres problèmes qui m'inquiétaient et qui transparaissent dans le Matou. Les problèmes politiques que vit le Québec, par exemple. Ça s'est fait spontanément. Ce n'est pas un hasard mais, en même temps, ce n'est pas le fruit d'un froid calcul que Florent Boissonneault soit associé à Len Slipskin et que leur association tourne mal. C'est une espèce de - je ne dirais pas de «symbole" parce que je n'écris pas de littérature à symboles - mais c'est une espèce de cri que je lançais.

Pour moi, un romancier c'est un peu comme un ami ou un voisin qui arriverait devant moi et me dirait: Écoute, il m'est arrivé une histoire absolument extraordinaire; il faut que je te la raconte. Le mot "extraordinaire» est important. Il signifie, au sens premier: hors de l'ordinaire, de la quotidienneté. Des romans comme l'Étranger ou Madame Bovary essaient de transmettre l'impression de la quotidienneté. Mais pas le Matou, les Têtes à Papineau ou même la Grosse Femme d'à côté est enceinte. Je ne suis pas un expérimental. J'écris d'une façon... comment pourrais-je dire? je n'aime pas le mot «traditionnel», parce que ça a un côté vieille pantoufle, poussiéreux. J'essaie d'écrire d'une façon claire et efficace. Je travaille à l'intérieur de cette écriture dite traditionnelle. Prenons une phrase, n'importe laquelle... La maison brûlait, par exemple. C'est très "traditionnel». Tu as un article, tu as un sujet, tu as un verbe. C'est comme ça. Des dizaines de millions de personnes font de pareilles phrases à longueur de journée. Tout le monde, quoi. Mais ce que j'essaie d'ajouter à ce genre de phrases, c'est de la couleur, un rythme, des comparaisons nouvelles. J'essaie d'éviter les clichés, j'essaie de trouver un ton qui corresponde à ma voix à moi. J'essaie d'être Yves Beauchemin, en toute simplicité.

V. \& I. - Comment ce roman se distingue-t-il de la production québécoise habituelle?

* Du sommet d'un arbre, Montréal, Québec/Amérique, 1986, 139 p. (NDLR). 
Y. Beauchemin - C'est une question encore plus traîtresse. D’abord, je ne suis pas critique, ni professeur de littérature. Donc, je suis assez loin de ce qui s'écrit au Québec. On écrit je nẹ sais pas combien de dizaines de romans chaque année au Québec. Peut-être cent cinquante ou deux cents. Pour moi, la comparaison est à peu près impossible à faire. Je ne lis pas beaucoup, parce que j'écris. Et comme je suis impliqué dans beaucoup de choses, j’ai peu de temps. Évidemment, je lis chaque jour, au moins une heure ou deux par jour, mais ça ne se compare pas avec l'horaire d'un véritable amateur de lecture. Qu'est-ce qui distingue le Matou de la production québécoise actuelle? Je pourrais vous répondre d'une façon superficielle. Sa longueur, peut-être. C'est un des plus gros romans qui ait été écrit au Québec, depuis quelques années. C'est un roman qui se démarque aussi du joual, je pense. Il y a une utilisation partielle du joual dans le livre, surtout dans le cas des personnages de Monsieur Émile et de Rosario Gladu. Mais ce n'est pas un roman joualisant. C'est un roman également qui, au niveau des dialogues, a essayé de trouver un compromis, de faire un mariage entre un parler régionaliste et le français international. Entre un français québécois à tendance folklorique et complaisante et le français international, aseptisé, insipide. Le Matou se distingue de ces deux courants-là. Au niveau de l'atmosphère, des personnages et de la facture de l'intrigue, je pense que le roman s'inscrit dans un courant nord-américain et non européen. J'aime beaucoup la littérature américaine, à cause de son côté concret, physique. Je pense à des romans comme ceux de Steinbeck, Hemingway, Dos Passos, Salinger. Des romans très près de la vie. J'adore ça. J'ai sûrement dû être influencé par ces écrivains-là. J'ai été influencé également, au niveau de la structure, par des romans classiques, comme ceux de Dickens. Je pense que je fais partie d'une génération d’écrivains, avec Jacques Benoit, Jean-Yves Soucy, André Major, Jacques Poulin, Roch Carrier, Louis Caron, d'une génération d'écrivains qui est plus américaine et mọins européenne, moins française et plus continentale, remplie d'une espèce de joie de vivre qui s'exprime par une certaine sensualité, l'humour, un goût des choses concrètes et tactiles, qu'on remarque depuis longtemps dans beaucoup de livres américains. En général, c'est une littérature qu'on peut qualifier d'inquiète, mais qui n'est pas triste. Je pense qu'on peut dire cela du Matou également. Je suis un de ces écrivains-là, qui sont dans la trentaine ou la quarantaine, et qui ont comme autre caractéristique d'écrire dans une langue accessible. Comme Steinbeck pouvait être accessible et, en même temps, compter parmi les plus grands écrivains de sa génération. Pour eux, le plaisir d'écrire, le plaisir de lire et le plaisir de se faire lire ne sont qu'un seul et même plaisir. La seule chose qui distingue peut-être le Matou des autres livres, c'est qu'il a connu un succès commercial, énorme pour le Québec. Mais le succès, c'est une arme à deux tranchants, j'en suis bien conscient.

\section{V. \& I. - La publicité a-t-elle beaucoup aidé à la vente du roman?}

Y. Beauchemin - Bien sûr. J'ai eu un excellent éditeur, Jacques Fortin, p.d.g. de Québec-Amérique. C'est un homme d'affaires avisé. C'est à lui, entre autres, qu'on doit la publication du livre en France, qui, par ricochet, a beaucoup aidé à la vente du livre au Québec. Personne n'est prophète en son 
pays, c'est toujours la même vieille chose. Jacques Fortin avait déniché une attachée de presse, Hélène Pichette, qui a contacté Denis Héroux. Comme le livre connaissait un certain succès en France, Héroux s'est intéressé au livre et a décidé d'en faire un film, ce qui a aidé aux ventes du livre. C'est le phénomène de la boule de neige. Je dois le succès de mon livre à une foule de gens compétents et dynamiques.

\section{V. \& I. - Comment avez-vous choisi votre éditeur?}

Y. Beauchemin - De la même façon qu'un acheteur se choisit une auto: j'ai fait le tour des garages. Après avoir photocopié le manuscrit en dix copies et l'avoir fait lire par dix lecteurs différents qui m'ont ensuite soumis leurs commentaires et m'ont beaucoup aidé à améliorer mon livre, j'ai pris la version finale, je l'ai photocopiée en quatre ou cinq exemplaires et je suis allé la porter à quatre éditeurs différents, en indiquant à chacun que le livre se trouvait chez trois de leurs concurrents et que je voulais simplement connaître leur réaction. En d'autres mots, je marchandais, comme fait tout un chacun lorsqu'il s'agit de s'acheter un tapis, une Toyota ou un ordinateur. Cette façon de procéder fait que les éditeurs, au lieu de laisser traîner ton manuscrit sur leur bureau pendant six mois, le lisent dans les trois jours qui suivent. Les quatre éditeurs m'ont donc répondu très vite. Et les quatre réponses étaient positives. Donc, j’étais à même de choisir. J'ai chọisi l'éditeur qui me paraissait le plus dynamique et s'offrait à vendre mon livre le moins cher possible. Le premier éditeur m'a affirmé qu'il ne pouvait pas éditer mon livre en bas de vingt-cinq dollars l'exemplaire. Le quatrième l'éditait à quatorze dollars quatre-vingt-quinze $(14,95 \$)^{1}$ ! Vingt-cinq dollars, pour moi, c'était la mort; le prix devenait prohibitif. Après avoir mis sept ans à écrire, je voulais évidemment être lu. C'est bien légitime, n'est-ce pas? Pour obtenir le Matou, Jacques Fortin a choisi de prendre le risque de vendre le premier tirage - trois mille exemplaires, je crois - à prix coûtant. Il a misé sur le succès du livre. S'il n'en avait vendu que trois mille, avec un prix de détail si bas (pour un livre de six cents pages, sur papier de qualité, avec une couverture en quadrichromie), il n'aurait pratiquement pas fait un sou. Fortin est un excellent homme d'affaires. Un éditeur doit être un excellent homme d'affaires. Fortin est bien entouré et il connaît șon métier, contrairement à bien des éditeurs qui fonctionnent d'une façon tout à fait amateur, inconséquente et imprévoyante. Cette situation, je pense, est due en partie aux subventions gouvernementales qui font qu'un éditeur, dans beaucoup de cas, ne court à peu près aucun risque financier quand il édite un livre. Il est donc porté à éditer n'importe quoi, qu'il publie de n'importe quelle façon. Fortin ne voit pas le métier de eette façon. C'est un gars de la Beauce, entreprenant, dynamique, très sympathique, qui travaille dur et qui veut vendre ses livres. J'ai trouvé exactement ce que je cherchais.

\section{V. \& I. - Est-ce que la critique a contribué au succès du roman?}

Y. Beauchemin - Il faut se demander quelle influence exerce la critique sur le public lecteur. Je pense que deux facteurs jouent. Il y a le bouche à oreille qui, je pense, est très important et aussi les critiques. Quand on lit une 
critique dans un journal et que c'est un véritable éreintement du livre, ça ne porte pas à le lire, évidemment. Mais les sources d'information viennent aussi de nos amis, des gens qu'on connaît, etc. Et ça aussi est très important. Je pense que les deux ont été importants. Dans quelle mesure? C'est une question de sociologie littéraire à laquelle je ne peux pas répondre.

V. \& I. - Le fait que l'Enfirouapé ait gagné le prix France-Québec 1975 a-t-il contribué au succès du deuxième roman?

Y. Beauchemin - Cela m'a aidé auprès des éditeurs. Comme mon premier livre avait connu un certain succès, les trois ou quatre personnes influentes au Québec au niveau de la critique attendaient le deuxième livre, d'une certaine façon. Je pouvais compter également sur un meilleur accueil de la part des éditeurs. L'Enfirouapé avait connu du succès. Je ne sais pas, le tirage doit dépasser les dix mille ( 10000$)$ exemplaires maintenant. Ainsi, en arrivant avec mon annuaire téléphonique chez l'éditeur - le manuscrit était vraiment énorme - il y avait moins de chance que l'éditeur fasse un infarctus en voyant la grosseur du manuscrit et j'avais plus de chance d'être pris au sérieux. J'étais vraiment très gêné de présenter une pareille brique, d'autant plus que j'étais bien conscient que le format habituel des romans au Québec dépasse rarement les deux cents, trois cents pages. Le succès de l'Enfirouapé a aidé, c'est sûr. Ensuite, il y avait les lecteurs qui avaient lu mon premier roman et parmi eux, ceux qui l'avaient aimé. Quand ils ont vu mon nom, ils se sont peut-être rappelés vaguement - il y a sept ans qui séparent les deux parutions - le nom d'Yves Beauchemin et ça leur a peut-être donné le goût de prendre une chance de lire le Matou. Mais il y a un facteur très important qu'on oublie trop dans le succès du Matou - et c'est mon côté américain -, c'est le prix du livre. Comme je l'ai dit, j'ai choisi, moi, l'éditeur qui le publierait au plus bas prix possible. Il fallait éviter que le prix trop élevé du livre ne rebute le lecteur éventuel. D'abord, c'était un roman québécois. Or, pour un lecteur québécois moyen, un roman québécois est moins bon qu'un roman étranger. Et puis, on ne dépense pas quinze dollars tous les jours pour acheter des livres qui ne sont pas utilitaires ou dont on n'est pas absolument sûr. Je pense que le prix modéré du livre joue énormément. D'ailleurs, il n'a jamais augmenté malgré l'inflation. Donc, il est de moins en moins cher et ça, c'est très important.

V. \& I. - À votre avis, le succès en France a-t-il fait augmenter les ventes au Québec?

Y. Beauchemin - Probablement. Il y a eu l'émission Apostrophes qui est diffusée au canal 99 . Il y a la publicité que mon éditeur a faite en utilisant des extraits de la presse française. Ça a sûrement aidé. Cela a peut-être rassuré certaines personnes qui se disaient: Ouf! après tout, c'est seulement un auteur québécois, il ne doit pas être si bon que ça. Quand les critiques français emboîtent le pas, ça donne peut-être un peu plus de courage aux gens pour acheter ce gros bouquin. En général, c'est ce qui se passe.

V. \& I. - Sur quoi vos personnages sont-ils modelés? Croyez-vous que les lecteurs se retrouvent dans les personnages du roman? 
Y. Beauchemin - Ça dépend des personnages. Comme tous les romanciers, je m'inspire de la vie. Qu'il s'agisse des personnages ou de l'histoire. On ne crée rien à partir du néant. Il y a des traits de certains de mes personnages qui proviennent de personnes que je connais, que j'ai rencontrées, que j'ai vues pendant quelques minutes, d'autres que j'ai fréquentées pendant des années. Je pense, entre autres, au cuisinier Aurélien Picquot, à Monsieur Émile. Monsieur Émile est inspiré d'une demi-douzaine d'enfants que j'ai connus, certains que j'ai vus quelques secondes, d'autres pendant des mois ou des années. Il y a un amalgame qui se crée, qui se fait lentement en nous, inconsciemment. À un moment donné, un personnage sort de notre tête. On l'accepte ou on ne l'accepte pas. Mais habituellement, c'est le personnage qui nous accepte lui-même, ou qui ne nous accepte pas. Il y a des personnages qui sont plus ordinaires, plus courants que d'autres, comme Élise et Florent. Ils sont peu caricaturés. Évidemment, pour un lecteur "ordinaire», c'est plus facile de s'y retrouver. Aurélien Picquot, Rosario Gladu, Monsieur Émile, c'est beaucoup plus stylisé, expressionniste. À ce moment-là, l'identification du lecteur aux personnages est sûrement beaucoup plus restreinte. Mais enfin, on peut retrouver une certaine partie de soi-même dans certains personnages sans que ça soit au complet. Je ne pense pas qu'il y ait beaucoup de gens qui se retrouvent dans Ratablavasky. Il y a peut-être certains lecteurs qui se retrouvent dans Rosario Gladu, certains lecteurs particulièrement vulgaires ou communs. C'est difficile à évaluer pour un auteur.

V. \& I. - D'où vous est venue l'inspiration pour votre roman? Est-ce que vos souvenirs d'enfance vous influencent?

Y. Beauchemin - Mes souvenirs d'enfance, jusqu'ici, ne m'ont absolument pas guidé. Je n'ai jamais utilisé grand-chose de mon enfance dans les livres que j'ai faits. Je ne sais pas pourquoi. Je me suis souvent posé la question. On dirait que c'est une chose qui m'appartient et que je ne veux partager avec personne. Il y a d'autres romanciers qui sont comme ça. C'est peut-être aussi à cause de l'attitude que j'ai par rapport à la littérature. L'Enfirouapé et le Matou ne sont pas des romans écrits au «je». Ce ne sont pas des romans qui sont très autobiographiques. Ce ne sont pas des romans subjectifs. Ce sont des romans un peu - je dis ça en toute modestie - un peu à la Dickens. C'est-à-dire que ce sont des créations, des constructions imaginaires dans lesquelles j'ai mis beaucoup de moi-même, mais qui ne reflètent pas ma vie personnelle. Il y a beaucoup de moi dans tout ça, mais ce n'est pas par le biais de mes souvenirs personnels. Ce n'est pas comme ça que ça se passe. L'inspiration du roman, d'où m'est-elle venue? Elle m'est venue de plusieurs choses. Elle m'est venue, comme je l'ai dit tout à l'heure, de gens que je connaissais. Elle m'est venue de livres que j'ai beaucoup aimés. Quand on écrit un roman, on essaie de rivaliser avec les romanciers qu'on a lus et qu'on a beaucoup aimés. Quand j’écris, je me rappelle Balzac, Tolstoï, Flaubert, Dickens, et j'essaie de, non pas faire comme eux, mais aussi bien qu'eux. Je dis ça, encore une fois, en toute modestie. Donc, il y a une partie des choix qu'on fait lorsqu'on écrit un livre qui vient des livres antérieurs qu'on a lus et qu'on a aimés. Je ne m'efforce pas en écrivant, vous pensez bien, de faire plus ennuyant et plus fade que les auteurs que j'ai aimés. J'essaie de faire plus 
intéressant, s'il est possible. Habituellement, c'est impossible. Une partie de mon inspiration vient des auteurs que j'ai aimés et avec qui j'essaie de rivaliser en utilisant mes propres moyens. Une autre partie me vient de la vie. Entre autres, de mon séjour de vingt ans à Montréal. Une autre partie me vient de la musique. En effet, la musique classique me stimule énormément. J'en écoute beaucoup. Je la préfère presque à la lecture. Elle donne de l'intensité, de la profondeur, à ma vie. Le personnage de Ratablavasky m'est venu d'une chanson de Moussorgsky, que j'écoutais un jour. Pendant que j'écoutais cette chanson, qui s'appelle l'Air de la puce, chantée par Kim Borg, la basse norvégienne, le personnage de Ratablavasky, dans sa suite à l'hôtel Nelson, m'est apparu instantanément. Il m'a été directement inspiré par Moussorgsky. En général, l'inspiration me vient de ma vie, de mes souvenirs. Mais, comme je l'ai dit, tout ça n'est pas utilisé d'une façon intimiste et autobiographique. C'est reconstruit, modifié d'une façon souvent bizarre. J'aime les constructions amples. Le roman que je suis en train d'écrire risque d'être aussi long que le Matou. Un roman, pour moi, c'est une sorte de ville imaginaire avec des dédales de rues, de places, de ruelles où j’essaie de concurrencer le réel.

V. \& I. - Les éléments antifédéralistes et antianglais que quelques critiques ont trouvés dans le roman, ont-ils aidé à son succès au Québec?

Y. Beauchemin - En fait, vous me demandez: Est-ce que la majorité des lecteurs du Matou sont, premièrement, politisés et, deuxièmement, indépendantistes? J'ai l'impression que la majorité des gens ne s'intéressent pas à la politique. Est-ce que les lecteurs québécois s'intéressent plus à la politique que la moyenne des gens? Oui et non. Le Matou a connu une diffusion assez grande. Donc, ça veut dire qu'il a été lu par des personnes qui ne lisent habituellement pas de livres québécois. Jacques Godbout, il y a quelques années, disait que le nombre d'amateurs de littérature québécoise chez nous tourne autour de quarante mille (40000). En tout cas, chose certaine, c'est une minorité. Je pense que la majorité des lecteurs assidus de romans québécois doivent s'intéresser à la politique parce qu'en partant ils sont éveillés aux choses de l'esprit et sont plus sensibilisés à toutes sortes de valeurs. Est-ce que, pour le public en général, les éléments politiques de mon livre constituaient une source d'attrait? Je ne serais pas porté à le croire. De toute façon, ce n'était pas sur ça que je misais. Ce qui était important pour moi, c'étaient les personnages et l'histoire, je le répète encore une fois. "Antianglais", je trouve ça un peu fort. Ce serait plutôt "antifédéraliste" qu'autre chose, parce que je ne suis pas anglophobe. J'aime trop la littérature britannique et américaine - et la culture qui leur a donné naissance - pour être vraiment canglophobe. Il ne faut jamais oublier qu'au Québec ce n'est pas un problème ethnique, c'est un problème politique. Ça ne peut pas se comparer, par exemple, avec le problème des Noirs aux États-Unis, où il y a un problème racial à la base de tout. Québécois et Canadiens, nous sommes de la même race. De la même civilisation. On s'adonne aux mêmes loisirs. On conduit les mêmes automobiles. On bâtit les maisons de la même façon. je me vois mal antianglais, moi qui habite une maison victorienne et qui voudrais tellement qu'on préserve l'héritage architectural de Montréal qui est, en grande partie, victorien. De l'héritage du Régime français à Montréal il ne reste presque 
rien. Les maisons que j'aime, ce sont des maisons qui ont été construites pour de riches marchands anglais, pour des financiers anglais, construites par des architectes anglais. J'ai assimilé ça comme tout le monde. Une belle maison, c'est une belle maison. On se fiche pas mal d'où elle vient. Donc, je ne me considère pas antianglais. Regardez ma bibliothèque. Tous ces rayons que vous voyez contiennent des livres anglais. Quand je lis un livre d'un auteur anglophone, je le lis dans sa langue à lui. Ma mère vient de l'Ontario. Ma mère est une franco-ontarienne bilingue qui a été professeur d'anglais pendant une bonne partie de sa vie. C'est par elle, et en lisant des comıc books quand j'étais enfant à Clova, que j'ai appris à lire l'anglais. Lorsque j'avais l'âge de six ou sept ans, je lisais l'anglais et le français. Je ne sens pas d'éléments antianglais en moi, pour toutes sortes de raisons. J'aime trop la vie pour ça. Dans mon livre, il y a des Anglais, il y a des Russes, il y a des juifs, il y a toutes sortes de gens. Dans le roman que je suis en train d'écrire, un des personnages importants est un musicien d'origine tchécoslovaque qui s'est établi à Montréal. S'il y a quelque chose que je n'aime pas, c'est le sectarisme et l'intolérance. Je disais que j'aimais la musique classique. J'aime Carl Nielsen (il est danois); j’aime Chostakovitch (il est russe); j'aime Mahler (il est juif et autrichien): j'aime Britten (il est anglais); j'aime Verdi (il est italien). Pour moi, la nationalité, c'est à la fois important et insignifiant. Nous avons un problème politique au Québec et ça s'adonne que ce n'est pas avec les Turcs que nous avons ce problème, ni avec les SerboCroates, mais avec les Canadiens anglais. C'est à la suite d'événements historiques bien précis. Surtout parce que Louis XV était un esprit médiocre et que Madame de Pompadour, sa maîtresse, dirigeait la politique étrangère, alors qu'en Angleterre il y avait William Pitt, père et fils, qui étaient pas mal plus réalistes et futés que cette chère Madame de Pompadour. Ce qui fait que les Français, stupidement, ont perdu leur empire colonial en Amérique et tant pis pour eux. Mais c'est nous qui payons, ce sont nous, les otages. Et bien sûr, c'est une situation désagréable. Est-ce que ça veut dire pour ça qu'on est antianglais? Non. J'ai hâte d'aller voir Londres, j'adore prendre mes vacances aux États-Unis, j'aime bien Toronto et Ottawa. Mais quand des problèmes politiques se prẻsentent --, là, c'est autre chose. Qu'est-ce que vous voulez, c'est comme si quelqu'un me disait: Est-ce que, en général, vous aimez vos voisins? Je répondrais: Oui, en général, j'aime mes voisins. Mais, si un de mes voisins, à toutes les fois que j'allume la radio, téléphone à la police, je n'aimerais pas ce voisin, bien sûr. Quand vous dites «antifédéraliste", j'aime ça, parce que vous vous référez à une structure politique et non à une ethnie. Et puis, je suis un pacifiste, moi. Le racisme me fait vomir. Les gens de ma génération ont été parmi les premiers en contact avec les documentaires sur l'Allemagne nazie. Je me rappelle, entre autres, un film réalisé par Alain Resnais, Nuit et Brouillard, dans les années 50, un des premiers films sur les camps de concentration de l'Allemagne nazie. Je me le rappellerai toute ma vie. J'ai vu ce film-là en juin 1961 ou 1962. C'était à la fin de l'année scolaire. À ce moment-là, j'étais étudiant au collège de Joliette et j'étais allé avec un copain fêter la fin des examens. On était montés à Montréal pour se gaver de cinéma et faire bonne chère. Comme tous les étudiants, on n'avait pas beaucoup d'argent à l'époque mais on voulait se payer du bon 
temps. En sortant du film, j'ai fait une terrible indigestion. C'est la seule fois que j’ai fait une indigestion aiguë. J'ai été vraiment très, très malade. Voir des cadavres d'enfants et d'adultes poussés par des bulldozers, ç'avait été pour moi un choc épouvantable. Tout ce qui est basé sur des réalités physiques ou affectives, comme la race, la culture, et qu'on utilise comme sources de haine, ça me fait vomir, littéralement. Je ne peux pas accepter ça. C'est viscéral. Je ne le peux tout simplement pas. C'est d'ailleurs une des raisons pour lesquelles je suis pacifiste. Mais être pacifiste et être bonasse, ce sont deux choses! Il faut aussi se faire respecter. La meilleure chose qui puisse arriver au Canada, c'est qu'on en modifie les structures politiques pour que l'harmonie s'établisse enfin. Pour moi, le Canada, c'est un mariage qui a mal tourné, et on force les deux époux à cohabiter. Si les époux arrivaient à régler certains problèmes, ils deviendraient peut-être amis, comme il arrive si souvent dans les cas de séparation ou de divorce. En tout cas, on pourrait au moins s'entendre sur les enfants. Dans ce cas-ci, l'enfant, c'est le territoire géographique et ses ressources. C'est ce que j'attends avec impatience. Malheureusement, par les temps qui courent, il semble qu'on s'éloigne de la solution.

V. \& I. - Est-ce que la situation politique au Québec était propice au succès du roman? C'est-à-dire, l'amélioration de l'image qu'ont d'eux-mêmes les Québécois, la loi 101, etc., aidaient-elles les ventes? Et puis, en deuxième partie: vous avez dit que les écrivains nous renvoient l'image moins déprimante de nous moins déprimés (Le Devoir, 4 juillet 1981, p. 13). Cette image moins déprimante se répercute-t-elle dans le succès du Matou?

Y. Beauchemin - Je pense que c'est plutôt l'inverse qui s'est produit. Si Florent est un héros vainqueur, contrairement à tant de personnages québécois qui sont des perdants, c'est à cause du contexte, je pense. Je n'ai pas décidé ça moi-même. J'ai commencé l'écriture du Matou en 1974. Donc, j’ai vécu en écrivant le Matou, toutes les années où s'est faite la montée du Parti québécois, sa popularité sans cesse grandissante. En 1977, on a promulgué la loi 101; on vivait encore dans l'enthousiasme de l'accession au pouvoir du parti indépendantiste. Tout ça crée une atmosphère très stimulante qui doit se refléter, je pense, dans le Matou. Une fois que cette asmosphère-là existe, les livres sortent. Ce n'est pas un cas qui m'est particulier, d'ailleurs. Et puis tout ça est bien antérieur à 1974, d'une certaine façon. Quand le livre sort et qu'il exprime une certaine joie de vivre - la joie de vivre que je ressens habituellement -, il y a des lecteurs qui vibrent. En général, je ne pense pas que beaucoup d'entre eux se complaisent dans la tristesse, l'inertie, la nonchalance et la dépression. Donc, le milieu a préparé le Matou; et le Matou reflète le milieu. Vous dites que les écrivains nous renvoient l'image moins déprimante de nous moins déprimés. Oui. Cependant, le Québec a bien changé depuis 1981. Depuis le référendum, le Québec traverse de nouveau une phase morose, une phase grise, une phase triste. Pas plus que quiconque, je ne connais l'avenir, je ne sais pas où cette situation va nous mener. Est-ce que les années 1976 à 1981 seront considérées comme un chant du cygne, un chant du cygne comme celui qu'a connu la Louisiane à la fin du XIXe siècle? En Louisiane, il $y$ avait une vie culturelle extrêmement brillante vers la fin du XIX ${ }^{e}$ siècle, 
jusqu’à la guerre de Sécession. Puis, ça s'est étiolé. Deux générations plus tard, il n'en restait plus rien. Est-ce que c'est ce qui est en train de nous arriver? Je n'en sais rien. Ce serait épouvantable. L'assimilation, c'est toujours une possibilité historique. La vie française en Amérique est terriblement fragile: nous représentons deux pour cent de la population nord-américaine! Le bilinguisme, c'est du cya nure de potassium pour nous, car il s'ajoute "par l'intérieur" à l'immense pression culturelle qu'exerce l'anglais sur notre minuscule collectivité. En Amérique, le bilinguisme a toujours été la phase préparatoire à l'unilinguisme anglais. Si le Québec devenait majoritairement bilingue dans son fonctionnement économique et socio-culturel, il serait comme un morceau de sucre dans un gallon de café. ça donnerait du café même pas sucré, voilà tout. Les sociétés et les pays ne sont pas immuables: ils peuvent disparaître. On retrouve ensuite leurs dépouilles dans les musées.

V. \& I. - Que pensez-vous de l'épithète d'antiféministe dont certaines personnes vous ont taxé?

Y. Beauchemin - Plusieurs femmes m'ont parlé du prétendu antiféminisme dans le Matou. Ça tourne essentiellement autour du caractère d'Élise. Élise est une femme que je pourrais qualifier de traditionnelle, comme il y en a beaucoup au Québec, comme il y en a beaucoup en Angleterre, en Australie, en France, etc. Ce n'est pas une militante féministe et les militantes féministes ne lui ont jamais pardonné de ne pas être militante féministe. Le couple d'Elise-Florent est un couple traditionnel, où l'homme, Florent, est le principal preneur de décisions au niveau... professionnel. Je ne dis pas au niveau affectif, parce que dans ce domaine, il est vraiment très inadéquat. Florent est ambitieux, il veut devenir riche, il fait des projets et Élise le suit. Elle ne le suit pas les yeux fermés. Elle le suit en le critiquant, elle le suit parfois, d'ailleurs, en lui lançant des assiettes par la tête. Mais Florent est un peu macho. Florent Boissonneault n'est pas un personnage particulièrement estimable. C'est un homme d'assez petite envergure, plutôt moyen, assez limité. Il ne possède pas la richesse affective d'Élise. Êlise est beaucoup plus humaine. C'est à cause d'elle que Monsieur Émile réussit à s'intégrer dans le couple. Pas à cause de Florent. Florent l'aime bien, il va même finir par s'attacher à lui, mais il est trop pris par ses affaires. Florent est un calculateur; il est un peu menteur aussi, ça ne lui déplaît pas parfois de mener double vie. Au point de vue moral, ce n'est pas un personnage particulièrement estimable. Élise l'est beaucoup plus. Élise est plus sincère, plus entière, plus sensible. À ce point de vue-là, Élise domine. Ceci dit, elle reste une femme traditionnelle et pour elle, une des choses importantes dans la vie, c'est d'avoir des enfants. Pour elle, la vie intime, la vie de foyer sont très importantes. C'est une valeur qui prime. Il ne faut pas oublier qu'on est en train de revenir à ça, tout le monde, les hommes et les femmes, après la grande vague féministe. Les femmes ont fait certains acquis, parce que leur situation antérieure était vraiment inacceptable. La parité de salaires est en train de se gagner. La possibilité d'avoir le même emploi qu'un homme - je parle pour le Québec - est en train de se gagner. La preuve de ça, c'est que les troubles pathologiques féminins tendent à ressembler à ceux de l'homme: haute pression, pro- 
blèmes circulatoires, cancer du poumon, troubles cardiaques, stress, etc. La femme est en train d'occuper une place équivalente à celle de l'homme et elle reçoit en partage ses problèmes physiques aussi. En même temps, depuis quelques années - c'est le vieux phénomène du balancier - , on est aussi en train de redécouvrir, après cette période de combat, que lorsqu'on veut élever des petits humains, le milieu qu'on leur donne est très important pour leur bonheur, présent et futur, et pour le bonheur du couple. On ne peut pas comme ça, dire, lorsqu'on a des enfants, qu'on soit homme ou femme, que la carrière professionnelle ou l'argent ou le confort doit l'emporter sur tout, sur n'importe quoi. D'après moi, c'est une attitude inhumaine, myope et très mesquine. Il y a une espèce de réajustement et un nouveau partage des tâches entre l'homme et la femme - en tout cas, chez les gens de ma classe sociale - qui sont en cours depuis plusieurs années. Moi, quand je reviens chez nous, ce n'est pas pour m'asseoir dans mon fauteuil, pantoufles au pied, et lire le journal, la caricature habituelle qu'on fait de l'homme traditionnel. Je m'occupe des enfants quand je suis à la maison autant que ma femme. Il y a un partage des tâches, quoi. Les critiques antiféministes qu'on a faites du livre, je ne les ai pas tellement prises au sérieux, parce que je sentais que, premièrement, les personnages qui apparaissent dans l'esprit d'un romancier ne lui demandent pas la permission pour le faire. Si le personnage d'Élise est apparu dans mon imagination, c'est probablement parce que ma mère était une femme assez traditionnelle, comme probablement votre mère aussi. Donc, le modèle que j'ai eu sous mes yeux pendant toute mon enfance était traditionnel. Moi-même, j'ai quarante-trois ans, et je ne peux me qualifier de marginal. J'ai évolué comme tout le monde, bien sûr. J'espère que je ne suis pas trop obtus. Mais je ne prétends pas être à la fine pointe de la façon de vivre des mâles en Occident. Je suis un homme de ma génération, voilà tout. J'ai quarante-trois ans, je n'ai pas vingt ans. Je fais une vie assez simple, assez réglée. Ca a donné Yves Beauchemin et c'est Yves Beauchemin qui écrit. Pour moi, ça n'aúrait pas été très sincère de fabriquer une femme qui aurait été différente d'Élise, parce que, au moment où j'ai pondu le premier jet de mon livre en 1974, les femmes que je connaissais et que je «sentais», étaient des femmes plutôt traditionnelles. Dans le prochain livre, ce sera peut-être différent.

V. \& I. - Est-ce parce que les accusations d'antiféminisme vous auront influencé?

Y. Beauchemin - Non. J'ai eu l'idée de mon troisième livre en 1975 ou 1976. Mon personnage principal est inspiré d'une femme de ménage que j’ai connue à Radio-Québec - on va me reprocher, je suppose, d'aller chercher mes personnages féminins dans des milieux trop modestes. J'étais devenu très ami avec cette femme, qui avait une cinquantaine d'années, et que je trouvais particulièrement pittoresque et colorée. C'était une espèce de force de la nature. Deuxièmement, il y a femmes et femmes dans le Matou. Il y a des femmes comme Élise, assez traditionnelles. Par contre, il y a la tante Jeunehomme qu'on ne peut certainement pas classer dans la même catégorie! La tante Jeunehomme, c'est une femme forte, une femme d'affaires. Les gens vont dire: Ah oui, tu l'as faite masculine! Elle n'est pas masculine. Il y a 
toutes sortes de femmes, vous le savez bien. Je peux vous jurer que l'institutrice qui m'a enseigné n'était absolument pas douce et effacée. La femme douce et effacée, c'est un cliché bébête. Il y a toutes sortes de femmes. Il y en a qui donnent des coups de poing sur la table. Et d'autres qui se pomponnent six heures par jour. D'autres qui font les deux. J'ai une cousine qui fait des journées de dix-huit heures en sifflotant. Moi, j'aurais la langue comme ça. Évidemment, il y a des conditions objectives dans la société qui jouent au détriment de la femme et à l'avantage de l'homme. Comme je l'ai dit, c'est une histoire que j'écrivais, je n'avais rien à prouver. Donc, ce personnage-là m'est venu comme ça et je l'acceptais comme tel. Ça a déplu aux féministes bien sûr, qui auraient voulu un personnage puissant, une supergirl. Je n'ai rien à répondre à ça.

V. \& I. - Que pensez-vous de la citation suivante: les snobs (...) disent que si tant de monde se retrouve dans un roman, c'est que le roman est médiocre (La Presse, 8 janvier 1983, p. C-3)?

Y. Beauchemin - Je pense que c'est une citation d'une interview que j'ai donnée à La Presse. Bien sûr, il ne s'agit pas de mon opinion à moi. Je suis de l'avis opposé. C'est toute la question de l'élitisme et du snobisme. Le Matou a connu un grand succès populaire. Ce genre de succès est une arme à deux tranchants - cliché! En sa présence, il y a deux réactions possibles. Chez la plupart des gens, ça stimule leur curiosité pour l'œuvre et, dans bien des cas, ça les incite à la lire. Pour d'autres - une minorité - , ça les porte à croire que si l'œuvre plaît à tant de monde, c'est qu'elle n'est pas bonne. Ils établissent l'équation suivante: la plupart des gens sont imbéciles; si un livre connaît du succès auprès de beaucoup de gens, c'est que le livre est imbécile! Or, c'est souvent vrai. Non pas que la plupart des gens sont imbéciles, mais que les livres faciles et plats attirent souvent beaucoup de lecteurs. Tout ce que j'espère, c'est que mon livre se trouve dans la bonne catégorie! Effectivement, les œuvres à grande diffusion sont habituellement du même niveau que le public auquel elles s'adressent - c'est-à-dire assez moyennes, ou parfois même carrément mauvaises. Mais cela souffre des exceptions. Le lecteur qui s'est délecté d'un livre facile et plat peut aussi se passionner pour une oeuvre accessible et d'excellente qualité. Sinon, comment expliquer la popularité de certains chefs-d'œuvres? En musique on pense à Beethoven et à Chopin. Ce sont des compositeurs qui plaisent à beaucoup de gens, et non pas seulement aux connaisseurs. En littérature, on pense, par exemple, à Tolstoï, à Dickens, à Victor Hugo, à Steinbeck. Evidemment, je ne suis pas en train de me classer parmi eux! Toutes ces catégorisations sont assez extérieures pour moi à l'acte d'écrire. Mais j'espère en tout cas, faire partie du groupe des écrivains honnêtes et consciencieux, qui respectent leur art et leur public.

V. \& I. - Selon Clotilde Painchaud (Grimoire, avril 1983, p. 8), vous avez été comparé à Paul Féval et à Guy des Cars, et puis, selon Gabrielle Poulin (Le Droit, 8 août, p. 14), à Nicolas Gogol. Que pensez-vous de ces comparaisons?

Y. Beauchemin - La comparaison avec Paul Féval à laquelle fait allusion Clotilde Painchaud n'exprime pas son opinion personnelle. Elle exprime 
plutôt celle de Patrick Grainville, auteur d'un roman qui a connu beaucoup de succès: les Flamboyants. Patrick Grainville est en même temps critique pour une publication du nom de VSD. Son article est paru le premier avril 1982. Le passage en question se lit comme suit: C'est alerte et pittoresque, il y a foule d'idées marrantes, parfois c'est un peu longuet. Cependant ni Rabelais ni Céline ne sont au rendez-vous. C'est du Féval et c'est déjà pas mal. Un excellent roman populiste donc! Ce sont les opinions de M. Grainville et je les respecte, comme on dit en pareille occasion. Ce n'est pas abominablement méchant, juste un peu condescendant, avec un soupçon de mépris. Je lui souhaite de meilleurs critiques que lui-même, c'est tout. Mais pour revenir à votre question, je ne sais trop quoi vous répondre. Paul Féval m'a donné beaucoup de plaisir quand j'étais jeune. Il est redevenu à la mode récemment parce que la littérature populiste, depuis quelques années, connaît une certaine vogue. Est-ce que je suis un petit Paul Féval québécois? En tout cas, je ne possède pas sa fécondité! Si mes lecteurs éprouvent autant de plaisir à me lire que j'en ai eu lorsque enfant je lisais Paul Féval, eh bien tant mieux pour eux. Quant à Guy des Cars, j’avoue ne pas l'avoir tellement fréquenté. Les débuts de romans que j'ai lus de lui ne m'ont pas donné le goût de continuer. J'espère être un cran ou deux au-dessus de lui, mais ce n'est pas moi qui décide. En ce qui a trait aux comparaisons avec Nicolas Gogol, nous nous trouvons dans l'extrême opposé. De la critique plutôt méchante à la louange excessive. Gogol est un génie et un de mes écrivains préférés. Quand j'entends de pareils compliments, je sens comme l'écroulement d'un mur de briques sur moi. La comparaison avec Nicolas Gogol vient peut-être du fait qu'il se trouve présent dans le Matou. Un des personnages de mon livre, en effet, cherche à reconstituer la deuxième partie des Âmes mortes, détruite par Gogol. J'aimerais bien être un écrivain du calibre de Nicolas Gogol, mais je crains fort qu'il faille me contenter d'être Yves Beauchemin.

\section{V. \& I. - Comment travaillez-vous?}

Y. Beauchemin - J'écris chaque jour. Cette habitude de régularité m'est venue de l'obligation d'intégrer l'écriture à mon travail quotidien. Je suis recherchiste, journaliste, si vous voulez, à Radio-Québec. J'écris pendant l'heure du dîner - entre midi et une heure et demie - et après cinq heures, entre cinq heures et six heures... six heures et demie. C'est mon métier qui m'impose une discipline. Certains se demandent: Comment l'inspiration descend-elle comme ça à heure fixe? Voilà une attitude bien romantique face à l'écriture! Lorsqu'on a une histoire en cours, ce n'est pas tellement d'inspiration qu'on a besoin chaque jour, mais d'une période de réchauffement pour se replonger dans le récit qu'on a laissé la veille ou quelques heures plus tôt. Dans la rédaction d'un roman, je sais toujours où je m'en vais, car j'ai rédigé au préalable des résumés, des descriptions caractérielles. Le dernier résumé compte environ cinquante pages. Voilà mon inspiration pour chaque jour. La période de réchauffement, elle, celle qui nous «met en transe» afin d'écrire, est plus capricieuse et problématique. Si tu as une heure et demie chaque midi pour travailler, il ne faut pas que ta période de réchauffement te prenne une heure, évidemment... Parfois, hélas, cela arrive. Mais habituellement, quand je cesse d'écrire, ce n'est pas parce que je n'ai plus rien à dire, c'est parce 
qu'on n'a plus le temps de le dire. Donc, en terminant, j'ai déjà des idées en réserve pour le lendemain. Souvent, je me mets à l'œuvre immédiatement après avoir glissé la feuille sous le rouleau de la machine à écrire. Les écrivains développent des automatismes, comme les joggeurs ou les plombiers. Écrire n'a rien en soi de particulièrement magique ou sublime. C'est une activité intellectuelle honorable, comme bien d'autres.

\section{V. \& I. - Dans quelles conditions matérielles écrivez-vous le mieux?}

Y. Beauchemin - Comme pour n'importe quelle activité, je pense, qu'il s'agisse d'un bon repas au restaurant, de faire l'amour, ou de réparer un moteur délicat, il ne faut pas être sous l'effet de soucis trop accablants. Si je suis soucieux, malade ou trop fatigué, j'écris peu ou mal ou les deux à la fois. Si je me couche à une heure du matin, je peux être sûr que ma journée d'écriture du lendemain est flambée. Donc l'écriture, comme n'importe quel métier, suppose une certaine discipline. À moins d'être une force de la nature, on ne peut pas mener une vie de bâton de chaise et être en même temps un bon professeur ou notaire ou électricien, n'est-ce pas? Dans le cas de l'écriture, c'est encore plus important d'être en bonne forme physique parce que, contrairement à la plupart des occupations professionnelles, il n'y a pas de stimuli extérieurs qui viennent nous mettre en branle. Si vous êtes, par exemple, gérant de banque, et que vous arrivez au travail un matin complètement crevé après une indigestion qui vous a gâché toute la nuit, vous devrez quand même faire face à des clients et à des employés qui vous attendent et qui exigent de vous toutes sortes de choses. Ça vous met forcément en condition pour donner un certain rendement. Mais quand je m'installe seul dans une pièce silencieuse devant une machine à écrire, je suis mon propre patron. Il n'y a personne qui a besoin de mes livres, il n'y a personne qui a besoin des livres de personne en particulier. Mon travail ne répond à aucun besoin extérieur pressant et impérieux. Alors, si je ne suis pas en forme, si je ne suis pas capable de m'autostimuler, si j'ai la tête pleine de ouate, c'est foutu. Oh! et puis il y a aussi, bien sûr, les coups de sonnette, les appels téléphoniques...

\section{V. \& I. - Mais revenons à votre travail d'écrivain. Comment cela se passe-t-il exactement?}

Y. Beauchemin - J'utilise la machine à écrire. Je ne peux écrire très longtemps avec un crayon ou une plume, car étant un gaucher qu'on a forcé à écrire de la main droite à l'école primaire, j'ai acquis une fausse position de la main qui devient rapidement douloureuse. D'ailleurs, écrire à la machine a un grand avantage; ça donne vaguement l'impression que le texte est publié - les caractères sont imprimés et ça distancie un peu le texte, qui devient plus facile à corriger. Et puis, pour écrire, j'ai besoin de silence. Je ne suis pas comme Anton Dvorák qui était capable de composer sur sa table de cuisine, parmi ses enfants. Il trouvait important que la vie familiale se déroule autour de lui. Mais la plupart des créateurs aiment, je pense, se retirer; c'est une question de concentration. Je produis toujours trois ou quatre versions successives d'un livre. J'essaie d'écrire mon premier jet le plus rapidement pos- 
sible. Je travaille d'une façon assez approximative et débraillée, afin de ne pas perdre le rythme.

V. \& I. - La dernière question: quelles sont, en bref, selon vous, les raisons du succès du roman? Quelle est la «recette» de votre prochain best-seller?

Y. Beauchemin - Je n'ai pas de recette. Personne n'a de recette. Et je n'aime pas tellement le mot best-seller. En général, les best-sellers ne sont pas de très bon livres. Ils sont destinés, la plupart du temps, à une consommation-éclair, puis à un oubli rapide. Le mot best-seller possède une connotation industrielle et commerciale. Je suis très conscient que la littérature repose sur des assises industrielles et commerciales - c'est fondamental - mais en même temps, elle vise plus haut que le profit, elle vise à rendre les hommes et les femmes meilleurs et plus heureux, elle vise au fond à l'éternité. À l'intérieur de chaque écrivain, il y a un visionnaire, un idéaliste. Il n'y aurait pas de vraie littérature si on ne trouvait au fond de lui qu'un homme d'affaires ou un amuseur. Vous me demandez d'énumérer les raisons du succès de mon roman? Il y en a plusieurs, et certaines que je ne connais pas. Énumérons: son prix modéré, compte tenu de sa grosseur; sa présentation attrayante (l'illustration de la couverture est de ma sœur Anne); la bonne qualité de la reliure et du papier; le bon accueil de la critique; l'accessibilité du roman (on est loin de la littérature expérimentale et cérébrale); le rythme de l'action, peut-être; l'humour que j'ai essayé d'y mettre; les personnages colorés, que je voulais fortement typés, même s'ils n'apparaissent que pendant une demi-page (ce souci me vient de la lecture de Tourguéniev); et, facteur très important: l'excellence de la mise en marché. Fortin est un éditeur compétent, audacieux; c'est à lui que je dois la diffusion de mon livre à l'étranger; c'est lui et Claude Choquette, son agent, qui ont veillé à ce que les traductions voient le jour. Mon premier roman n'avait pas été aussi bien servi. A cause sans doute de l'exiguïté de notre marché, bien des maisons d'édition ont conservé un caractère artisanal et amateur. Et puis enfin, il y a les impondérables: la chance, la parution au bon moment, la correspondance presque fortuite avec les goûts d'un public. Est-ce que cela va se reproduire avec mon prochain livre? Je n'en sais rien. Il n'y a pas de recette-pour créer de bons personnages ou une intrigue passionnante, il n'y a pas de recette pour trouver des images originales, des reparties piquantes ou des comparaisons amusantes. Il faut que tout ça soit contenu dans le germe même de l'histoire, ce qui dépend très peu de la volonté de l'écrivain. Alors, comme les lecteurs, comme l'éditẹur, moi aussi je suis placé devant l'inconnu.

1. Selon Claude Martin ( «Comme des petits pains chauds. Essai d'économie industrielle du best-seller en français au Québec", Communication Information, automne 1985, vol. 7 , no 3 , p. 114), quatọze dollars quatre-vingt-quinze $(14,95 \$)$ est le prix idéal d'un best-seller. 


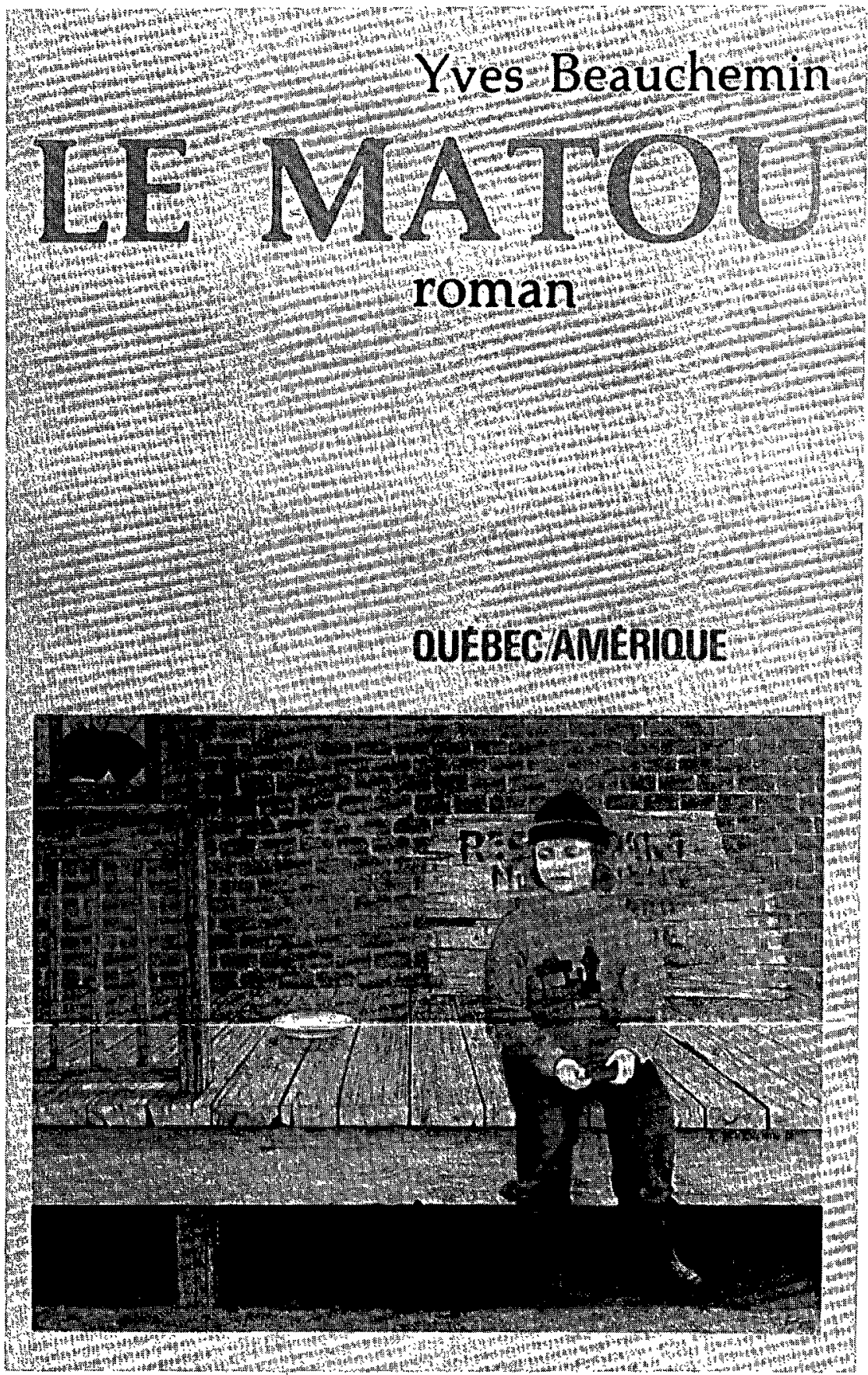

\section{Première édition.}

